\title{
Strong Convergence Results for Equilibrium Problems and Fixed Point Problems for Multivalued Mappings
}

\author{
J. Vahidi, ${ }^{1}$ A. Latif, ${ }^{2}$ and M. Eslamian ${ }^{3}$ \\ ${ }^{1}$ Department of Mathematics, Iran University of Science and Technology, Behshahr, Iran \\ ${ }^{2}$ Department of Mathematics, King Abdulaziz University, P.O. Box 80203, Jeddah 21589, Saudi Arabia \\ ${ }^{3}$ Department of Mathematics, Science and Research Branch, Islamic Azad University, Tehran, Iran
}

Correspondence should be addressed to A. Latif; latifmath@yahoo.com

Received 21 July 2013; Accepted 10 October 2013

Academic Editor: Mohamed Amine Khamsi

Copyright (C) 2013 J. Vahidi et al. This is an open access article distributed under the Creative Commons Attribution License, which permits unrestricted use, distribution, and reproduction in any medium, provided the original work is properly cited.

Using viscosity approximation method, we study strong convergence to a common element of the set of solutions of an equilibrium problem and the set of common fixed points of a finite family of multivalued mappings satisfying the condition $(E)$ in the setting of Hilbert space. Our results improve and extend some recent results in the literature.

\section{Introduction}

Let $H$ be a real Hilbert space with inner product $\langle\cdot, \cdot\rangle$ and norm $\|\cdot\|$. Let $C$ be a nonempty closed convex subset $H$. A subset $C \subset H$ is called proximal if, for each $x \in H$, there exists an element $y \in C$ such that

$$
\|x-y\|=\operatorname{dist}(x, C)=\inf \{\|x-z\|: z \in C\} .
$$

A single-valued mapping $T: C \rightarrow C$ is said to be nonexpansive, if

$$
\|T x-T y\| \leq\|x-y\|, \quad \forall x, y \in C .
$$

Let $P_{C}$ be a nearest point projection of $H$ into $C$; that is, for $x \in H, P_{C} x$ is a unique nearest point in $C$ with the property

$$
\left\|x-P_{C} x\right\|:=\inf \{\|x-y\|: y \in C\} .
$$

We denote by $C B(C), K(C)$, and $P(C)$ the collection of all nonempty closed bounded subsets, nonempty compact subsets, and nonempty proximal bounded subsets of $C$ respectively. The Hausdorff metric $H$ on $C B(H)$ is defined by

$$
H(A, B):=\max \left\{\sup _{x \in A} \operatorname{dist}(x, B), \sup _{y \in B} \operatorname{dist}(y, A)\right\},
$$

for all $A, B \in C B(H)$.
Let $T: H \rightarrow 2^{H}$ be a multivalued mapping. An element $x \in H$ is said to be a fixed point of $T$, if $x \in T x$ and the set of fixed points of $T$ is denoted by $F(T)$.

A multivalued mapping $T: H \rightarrow C B(H)$ is called

(i) nonexpansive if

$$
H(T x, T y) \leq\|x-y\|, \quad x, y \in H
$$

(ii) quasi-nonexpansive if $F(T) \neq \emptyset$ and $H(T x, T p) \leq \| x-$ $p \|$ for all $x \in H$ and all $p \in F(T)$.

Recently, García-Falset et al. [1] introduced a new condition on single-valued mappings, called condition $(E)$, which is weaker than nonexpansiveness.

Definition 1. A mapping $T: H \rightarrow H$ is said to satisfy condition $\left(E_{\mu}\right)$ provided that

$$
\|x-T y\| \leq \mu\|x-T x\|+\|x-y\|, \quad x, y \in H .
$$

We say that $T$ satisfies condition $(E)$ whenever $T$ satisfies $\left(E_{\mu}\right)$ for some $\mu \geq 1$.

Recently, Abkar and Eslamian [2,3] generalized this condition for multivalued mappings as follows. 
Definition 2. A multivalued mapping $T: H \rightarrow C B(H)$ is said to satisfy condition $(E)$ provided that

$$
H(T x, T y) \leq \mu \operatorname{dist}(x, T x)+\|x-y\|, \quad x, y \in H,
$$

for some $\mu \geq 1$.

It is obvious that every nonexpansive multivalued mapping $T: H \rightarrow C B(H)$ satisfies the condition $(E)$, and every mapping $T: H \rightarrow C B(H)$ which satisfies the condition $(E)$ with nonempty fixed point set $F(T)$ is quasi-nonexpansive.

Example 3. Let us define a mapping $T$ on $[0,3]$ by

$$
T(x)= \begin{cases}{\left[0, \frac{x}{3}\right],} & x \neq 3 \\ {[1,2]} & x=3 .\end{cases}
$$

It is easy to see that $T$ satisfies the condition $(E)$ but is not nonexpansive. Indeed, for $x, y \in[0,3), H(T x, T y)=\mid(x-$ $y) / 3|\leq| x-y \mid$. Let $x=0$ and $y=3$. Then $H(T x, T y)=$ $2 \leq 3=|x-y|$. If $x \in(0,3)$ and $y=3$, then, we have $\operatorname{dist}(x, T x)=2 x / 3$ and $\operatorname{dist}(y, T y)=1$; hence

$$
H(T x, T y)=2-\frac{x}{3} \leq 3-x+\frac{4 x}{3}=|x-y|+2 \operatorname{dist}(x, T x) .
$$

Thus, $T$ satisfies the condition $(E)$. However, $T$ is not nonexpansive; indeed for $x=3$ and $y=7 / 3, H(T x, T y)=$ $11 / 9>2 / 3=|x-y|$.

Let $\Psi: C \times C \rightarrow \mathbb{R}$ be a bifunction. The equilibrium problem associated with the bifunction $\Psi$ and the set $C$ is:

$$
\text { find } x \in C \text { such that } \Psi(x, y) \geq 0, \forall y \in C \text {. }
$$

Such a point $x \in C$ is called the solution of the equilibrium problem. The set of solutions is denoted by $E P(\Psi)$.

A broad class of problems in optimization theory, such as variational inequality, convex minimization, and fixed point problems, can be formulated as an equilibrium problem; see $[4,5]$. In the literature, many techniques and algorithms have been proposed to analyze the existence and approximation of a solution to equilibrium problem; see [6]. Many researchers have studied various iteration processes for finding a common element of the set of solutions of the equilibrium problems and the set of fixed points of a class of nonlinear mappings. For example, see [7-22].

Fixed points and fixed point iteration process for nonexpansive mappings have been studied extensively by many authors to solve nonlinear operator equations, as well as variational inequalities; see, for example, [23-28]. In the recent years, fixed point theory for multivalued mappings has been studied by many authors; see [29-40] and the references therein.

In this paper, using viscosity approximation method, we study strong convergence to a common element of the set of solutions of an equilibrium problem and the set of common fixed points of a finite family of multivalued mappings satisfying the condition $(E)$ in the setting of Hilbert space. Our results improve and extend some recent results in the literature.

\section{Preliminaries}

For solving the equilibrium problem, we assume that the bifunction $\Psi$ satisfies the following conditions:

(A1) $\Psi(x, x)=0$ for any $x \in C$;

(A2) $\Psi$ is monotone; that is, $\Psi(x, y)+\Psi(y, x) \leq 0$ for any $x, y \in C$;

(A3) $\Psi$ is upper-hemicontinuous; that is, for each $x, y, z \in$ C,

$$
\lim _{t \rightarrow 0^{+}} \sup \Psi(t z+(1-t) x, y) \leq \Psi(x, y) ;
$$

(A4) $\Psi(x,$.$) is convex and lower semicontinuous for each$ $x \in C$.

Lemma 4 (see [4]). Let $C$ be a nonempty closed convex subset of $H$ and let $\Psi$ be a bifunction of $C \times C$ into $\mathbb{R}$ satisfying (A1)-(A4). Let $r>0$ and $x \in H$. Then, there exists $z \in C$ such that

$$
\Psi(z, y)+\frac{1}{r}\langle y-z, z-x\rangle \geq 0 \quad \forall y \in C .
$$

Lemma 5 (see [6]). Assume that $\Psi: C \times C \rightarrow \mathbb{R}$ satisfies $(A 1)-(A 4)$. For $r>0$ and $x \in H$, define a mapping $S_{r}: H \rightarrow$ $C$ as follows:

$$
S_{r} x=\left\{z \in C: \Psi(z, y)+\frac{1}{r}\langle y-z, z-x\rangle \geq 0, \quad \forall y \in C\right\} .
$$

Then, the following hold:

(i) $S_{r}$ is single valued;

(ii) $S_{r}$ is firmly nonexpansive; that is, for any $x, y \in H$,

$$
\left\|S_{r} x-S_{r} y\right\|^{2} \leq\left\langle S_{r} x-S_{r} y, x-y\right\rangle ;
$$

(iii) $F\left(S_{r}\right)=E P(\Psi)$;

(iv) $E P(\Psi)$ is closed and convex.

Lemma 6 (see [41]). Let $H$ be a real Hilbert space. Then, for all $x, y, z \in H$ and $\alpha, \beta, \gamma \in[0,1]$ with $\alpha+\beta+\gamma=1$ one has

$$
\begin{aligned}
\|\alpha x+\beta y+\gamma z\|^{2}= & \alpha\|x\|^{2}+\beta\|y\|^{2}+\gamma\|z\|^{2} \\
& -\alpha \beta\|x-y\|^{2}-\alpha \gamma\|x-z\|^{2}-\beta \gamma\|z-y\|^{2} .
\end{aligned}
$$

Lemma 7. For every $x$ and $y$ in a Hilbert space $H$, the following inequality holds:

$$
\|x+y\|^{2} \leq\|x\|^{2}+2\langle y, x+y\rangle .
$$

Lemma 8 (see [42]). Let $\left\{a_{n}\right\}$ be a sequence of nonnegative real numbers, $\left\{\alpha_{n}\right\}$ a sequence in $(0,1)$ with $\sum_{n=1}^{\infty} \alpha_{n}=\infty,\left\{\gamma_{n}\right\}$ a sequence of nonnegative real numbers with $\sum_{n=1}^{\infty} \gamma_{n}<\infty$, and $\left\{\beta_{n}\right\}$ a sequence of real numbers with $\lim \sup _{n \rightarrow \infty} \beta_{n} \leq 0$. Suppose that the following inequality holds:

$$
a_{n+1} \leq\left(1-\alpha_{n}\right) a_{n}+\alpha_{n} \beta_{n}+\gamma_{n}, \quad n \geq 0 .
$$

Then, $\lim _{n \rightarrow \infty} a_{n}=0$. 
Lemma 9 (see [43]). Let $\left\{u_{n}\right\}$ be a sequence of real numbers that does not decrease at infinity, in the sense that there exists a subsequence $\left\{u_{n_{i}}\right\}$ of $\left\{u_{n}\right\}$ such that $u_{n_{i}}<u_{n_{i}+1}$ for all $i \geq 0$. For every sufficiently large number $n \geq n_{0}$, define an integer sequence $\{\tau(n)\}$ as

$$
\tau(n)=\max \left\{k \leq n: u_{k}<u_{k+1}\right\} .
$$

Then, $\tau(n) \rightarrow \infty$ as $n \rightarrow \infty$ and for all $n \geq n_{0}$,

$$
\max \left\{u_{\tau(n)}, u_{n}\right\} \leq u_{\tau(n)+1} .
$$

Lemma 10 (see [20]). Let $C$ be a closed convex subset of a real Hilbert space $H$. Let $T: C \rightarrow C B(C)$ be a quasi-nonexpansive multivalued mapping. If $F(T) \neq \emptyset$ and $T(p)=\{p\}$ for all $p \in$ $F(T)$. Then $F(T)$ is closed and convex.

Lemma 11 (see [20]). Let $C$ be a closed convex subset of a real Hilbert space $H$. Let $T: C \rightarrow P(C)$ be a multivalued mapping such that $P_{T}$ is quasi-nonexpansive and $F(T) \neq \emptyset$, where $P_{T}(x)=\{y \in T x:\|x-y\|=\operatorname{dist}(x, T x)\}$. Then, $F(T)$ is closed and convex.

Lemma 12 (see $[16,20])$. Let $C$ be a nonempty closed convex subset of a real Hilbert space $H$. Let $T: C \rightarrow K(C)$ be a multivalued mapping satisfying the condition $(E)$. If $x_{n}$ converges weakly to $v$ and $\lim _{n \rightarrow \infty} \operatorname{dist}\left(x_{n}, T x_{n}\right)=0$, then $v \in T v$.

\section{A Strong Convergence Theorem}

Theorem 13. Let $C$ be a nonempty closed convex subset of a real Hilbert space $H$ and $\Psi$ a bifunction of $C \times C$ into $\mathbb{R}$ satisfying $(A 1)-(A 4)$. Let $T_{i}: C \rightarrow C B(C)(i=1,2, \ldots, m)$ be a finite family of multivalued mappings, each satisfying condition (E). Assume further that $\mathscr{F}=\bigcap_{i=1}^{m} F\left(T_{i}\right) \bigcap E P(\Psi) \neq \emptyset$ and $T_{i}(p)=\{p\},(i=1,2, \ldots, m)$ for each $p \in \mathscr{F}$. Let $f$ be a $k$-contraction of $C$ into itself. Let $\left\{x_{n}\right\}$ and $\left\{u_{n}\right\}$ be sequences generated the following algorithm:

$$
\begin{aligned}
& x_{0} \in C \text {, } \\
& u_{n} \in C \text { such that } \Psi\left(u_{n}, y\right)+\frac{1}{r_{n}}\left\langle y-u_{n}, u_{n}-x_{n}\right\rangle \geq 0 \text {, } \\
& \forall y \in C \\
& y_{n, 1}=a_{n, 1} u_{n}+b_{n, 1} x_{n}+c_{n, 1} z_{n, 1} \text {, } \\
& y_{n, 2}=a_{n, 2} u_{n}+b_{n, 2} z_{n, 1}+c_{n, 2} z_{n, 2} \text {, } \\
& y_{n, 3}=a_{n, 3} u_{n}+b_{n, 3} z_{n, 2}+c_{n, 3} z_{n, 3} \\
& y_{n, m}=a_{n, m} u_{n}+b_{n, m} z_{n, m-1}+c_{n, m} z_{n, m} \text {, } \\
& x_{n+1}=\vartheta_{n} f\left(x_{n}\right)+\left(1-\vartheta_{n}\right) y_{n, m}, \\
& \forall n \geq 0,
\end{aligned}
$$

where $z_{n, 1} \in T_{1}\left(u_{n}\right), z_{n, k} \in T_{k}\left(y_{n, k-1}\right)$ for $k=2, \ldots, m$, and $\left\{a_{n, i}\right\},\left\{b_{n, i}\right\},\left\{c_{n, i}\right\},\left\{\vartheta_{n}\right\}$, and $\left\{r_{n}\right\}$ satisfy the following conditions:

(i) $\left\{a_{n, i}\right\},\left\{b_{n, i}\right\},\left\{c_{n, i}\right\} \subset[a, b] \subset(0,1), a_{n, i}+b_{n, i}+c_{n, i}=$ $1,(i=1,2, \ldots, m)$,

(ii) $\left\{\vartheta_{n}\right\} \subset(0,1), \lim _{n \rightarrow \infty} \vartheta_{n}=0, \sum_{n=1}^{\infty} \vartheta_{n}=\infty$,

(iii) $\left\{r_{n}\right\} \subset(0, \infty)$, and $\lim \inf _{n \rightarrow \infty} r_{n}>0$.

Then, the sequences $\left\{x_{n}\right\}$ and $\left\{u_{n}\right\}$ converge strongly to $q \in \mathscr{F}$, where $q=P_{\mathscr{F}} f(q)$.

Proof. Let $Q=P_{\mathscr{F}}$. It is easy to see that $Q f$ is a contraction. By Banach contraction principle, there exists a $q \in \mathscr{F}$ such that $q=P_{\mathscr{F}} f(q)$. From Lemma 5 for all $n \geq 0$, we have

$$
\left\|u_{n}-q\right\|=\left\|S_{r_{n}} x_{n}-S_{r_{n}} q\right\| \leq\left\|x_{n}-q\right\|
$$

We show that $\left\{x_{n}\right\}$ is bounded. Since, for each $i=1,2, \ldots, m$, $T_{i}$ satisfies the condition $(E)$ and we have

$$
\begin{aligned}
& \left\|y_{n, 1}-q\right\| \\
& \quad=\left\|a_{n, 1} u_{n}+b_{n, 1} x_{n}+c_{n, 1} z_{n, 1}-q\right\| \\
& \quad \leq a_{n, 1}\left\|u_{n}-q\right\|+b_{n, 1}\left\|x_{n}-q\right\|+c_{n, 1}\left\|z_{n, 1}-q\right\| \\
& \quad=a_{n, 1}\left\|u_{n}-q\right\|+b_{n, 1}\left\|x_{n}-q\right\|+c_{n, 1} \operatorname{dist}\left(z_{n, 1}, T_{1} q\right) \quad(22) \\
& \quad \leq a_{n, 1}\left\|u_{n}-q\right\|+b_{n, 1}\left\|x_{n}-q\right\|+c_{n, 1} H\left(T_{1} u_{n}, T_{1} q\right) \\
& \quad \leq a_{n, 1}\left\|u_{n}-q\right\|+b_{n, 1}\left\|x_{n}-q\right\|+c_{n, 1}\left\|u_{n}-q\right\| \\
& \quad \leq\left\|x_{n}-q\right\|, \\
& \left\|y_{n, 2}-q\right\| \\
& \quad=\left\|a_{n, 2} u_{n}+b_{n, 2} z_{n, 1}+c_{n, 2} z_{n, 2}-q\right\| \\
& \quad \leq a_{n, 2}\left\|u_{n}-q\right\|+b_{n, 2}\left\|z_{n, 1}-q\right\|+c_{n, 2}\left\|z_{n, 2}-q\right\| \\
& \quad=a_{n, 2}\left\|u_{n}-q\right\|+b_{n, 2} \operatorname{dist}\left(z_{n, 1}, T_{1} q\right)+c_{n, 2} \operatorname{dist}\left(z_{n, 2}, T_{2} q\right) \\
& \quad \leq a_{n, 2}\left\|u_{n}-q\right\|+b_{n, 2} H\left(T_{1} u_{n}, T_{1} q\right)+c_{n, 2} H\left(T_{2} y_{n, 1}, T_{2} q\right) \\
& \quad \leq a_{n, 2}\left\|u_{n}-q\right\|+b_{n, 2}\left\|u_{n}-q\right\|+c_{n, 2}\left\|y_{n, 1}-q\right\| \\
& \leq\left\|x_{n}-q\right\| .
\end{aligned}
$$

By continuing this process, we obtain

$$
\left\|y_{n, m}-q\right\| \leq\left\|x_{n}-q\right\|
$$


This implies that

$$
\begin{aligned}
& \left\|x_{n+1}-q\right\| \\
& \quad=\left\|\vartheta_{n} f x_{n}+\left(1-\vartheta_{n}\right) y_{n}-q\right\| \\
& \quad \leq \vartheta_{n}\left\|f x_{n}-q\right\|+\left(1-\vartheta_{n}\right)\left\|y_{n}-q\right\| \\
& \quad \leq \vartheta_{n}\left(\left\|f x_{n}-f q\right\|+\|f q-q\|\right)+\left(1-\vartheta_{n}\right)\left\|x_{n}-q\right\| \\
& \quad \leq \vartheta_{n} k\left\|x_{n}-q\right\|+\vartheta_{n}\|f q-q\|+\left(1-\vartheta_{n}\right)\left\|x_{n}-q\right\| \\
& \quad=\left(1-\vartheta_{n}(1-k)\right)\left\|x_{n}-q\right\|+\vartheta_{n}\|f q-q\| \\
& \quad \leq \max \left\{\left\|x_{n}-q\right\|, \frac{\|f q-q\|}{1-k}\right\} .
\end{aligned}
$$

By induction, we get

$$
\left\|x_{n}-q\right\| \leq \max \left\{\left\|x_{0}-q\right\|, \frac{\|f q-q\|}{1-k}\right\},
$$

for all $n \in \mathbb{N}$. This implies that $\left\{x_{n}\right\}$ is bounded and we also obtain that $\left\{u_{n}\right\},\left\{y_{n}\right\},\left\{f x_{n}\right\}$, and $\left\{z_{n, i}\right\}$ are bounded. Next, we show that $\lim _{n \rightarrow \infty} \operatorname{dist}\left(u_{n}, T_{i} u_{n}\right)=0$ for each $i \in \mathbb{N}$. By Lemma 6, we have

$$
\begin{aligned}
\left\|y_{n, 1}-q\right\|^{2} & \left\|a_{n, 1} u_{n}+b_{n, 1} x_{n}+c_{n, 1} z_{n, 1}-q\right\|^{2} \\
\leq & a_{n, 1}\left\|u_{n}-q\right\|^{2}+b_{n, 1}\left\|x_{n}-q\right\|^{2} \\
& +c_{n, 1}\left\|z_{n, 1}-q\right\|^{2} \\
& -a_{n, 1} b_{n, 1}\left\|x_{n}-u_{n}\right\|^{2}-a_{n, 1} c_{n, 1}\left\|u_{n}-z_{n, 1}\right\|^{2} \\
= & a_{n, 1}\left\|u_{n}-q\right\|^{2}+b_{n, 1}\left\|x_{n}-\mathrm{q}\right\|^{2} \\
& +c_{n, 1} \operatorname{dist}\left(z_{n, 1}, T_{1} q\right)^{2} \\
& -a_{n, 1} b_{n, 1}\left\|x_{n}-u_{n}\right\|^{2}-a_{n, 1} c_{n, 1}\left\|u_{n}-z_{n, 1}\right\|^{2} \\
\leq & a_{n, 1}\left\|u_{n}-q\right\|^{2}+b_{n, 1}\left\|x_{n}-q\right\|^{2} \\
& +c_{n, 1} H\left(T_{1} u_{n}, T_{1} q\right)^{2} \\
& -a_{n, 1} b_{n, 1}\left\|x_{n}-u_{n}\right\|^{2}-a_{n, 1} c_{n, 1}\left\|u_{n}-z_{n, 1}\right\|^{2} \\
\leq & a_{n, 1}\left\|u_{n}-q\right\|^{2}+b_{n, 1}\left\|x_{n}-q\right\|^{2} \\
& +c_{n, 1}\left\|u_{n}-q\right\|^{2} \\
& -a_{n, 1} b_{n, 1}\left\|x_{n}-u_{n}\right\|^{2}-a_{n, 1} c_{n, 1}\left\|u_{n}-z_{n, 1}\right\|^{2} \\
\leq & x_{n}-q\left\|^{2}-a_{n, 1} b_{n, 1}\right\| x_{n}-u_{n} \|^{2} \\
& -c_{n, 1}\left\|u_{n}-z_{n, 1}\right\|^{2} . \\
= &
\end{aligned}
$$

Applying Lemma 6 once more, we have

$$
\begin{aligned}
\| y_{n, 2} & -q \|^{2} \\
= & \left\|a_{n, 2} u_{n}+b_{n, 2} z_{n, 1}+c_{n, 2} z_{n, 2}-q\right\|^{2} \\
\leq & a_{n, 2}\left\|u_{n}-q\right\|^{2}+b_{n, 2}\left\|z_{n, 1}-q\right\|^{2}+c_{n, 2}\left\|z_{n, 2}-q\right\|^{2} \\
& -a_{n, 2} c_{n, 2}\left\|u_{n}-z_{n, 2}\right\|^{2} \\
= & a_{n, 2}\left\|u_{n}-q\right\|^{2}+b_{n, 2} \operatorname{dist}\left(z_{n, 1}, T_{1} q\right)^{2} \\
+ & c_{n, 2} \operatorname{dist}\left(z_{n, 2}, T_{2} q\right)^{2}-a_{n, 2} c_{n, 2}\left\|u_{n}-z_{n, 2}\right\|^{2} \\
\leq & a_{n, 2}\left\|u_{n}-q\right\|^{2}+b_{n, 2} H\left(T_{1} u_{n}, T_{1} q\right)^{2} \\
& +c_{n, 2} H\left(T_{1} y_{n, 1}, T_{2} q\right)^{2}-a_{n, 2} c_{n, 2}\left\|u_{n}-z_{n, 2}\right\|^{2} \\
\leq & a_{n, 2}\left\|u_{n}-q\right\|^{2}+b_{n, 2}\left\|u_{n}-q\right\|^{2}+c_{n, 2}\left\|y_{n, 1}-q\right\|^{2} \\
& -a_{n, 2} c_{n, 2}\left\|u_{n}-z_{n, 2}\right\|^{2} \\
\leq & \left\|x_{n}-q\right\|^{2}-a_{n, 2} c_{n, 2}\left\|u_{n}-z_{n, 2}\right\|^{2} \\
& -a_{n, 1} c_{n, 1} c_{n, 2}\left\|u_{n}-z_{n, 1}\right\|^{2}-a_{n, 1} b_{n, 1} c_{n, 2}\left\|x_{n}-u_{n}\right\|^{2} .
\end{aligned}
$$

By continuing this process we have

$$
\begin{aligned}
\left\|y_{n, m}-q\right\|^{2} & \left\|a_{n, m} u_{n}+b_{n, m} z_{n, m-1}+c_{n, m} z_{n, m}-q\right\|^{2} \\
\leq & a_{n, m}\left\|u_{n}-q\right\|^{2}+b_{n, m}\left\|z_{n, m-1}-q\right\|^{2}+c_{n, m}\left\|z_{n, m}-q\right\|^{2} \\
& -a_{n, m} c_{n, m}\left\|u_{n}-z_{n, m}\right\|^{2} \\
= & a_{n, m}\left\|u_{n}-q\right\|^{2}+b_{n, m} \operatorname{dist}\left(z_{n, m-1}, T_{m-1} q\right)^{2} \\
& +c_{n, m} \operatorname{dist}\left(z_{n, m}, T_{m} q\right)^{2}-a_{n, m} c_{n, m}\left\|u_{n}-z_{n, m}\right\|^{2} \\
\leq & a_{n, m}\left\|u_{n}-q\right\|^{2}+b_{n, m} H\left(T_{m-1} y_{n, m-2}, T_{m-1} q\right)^{2} \\
& +c_{n, m} H\left(T_{m} y_{n, m-1}, T_{m} q\right)^{2}-a_{n, m} c_{n, m}\left\|u_{n}-z_{n, m}\right\|^{2} \\
\leq & a_{n, m}\left\|u_{n}-q\right\|^{2}+b_{n, m}\left\|y_{n, m-2}-q\right\|^{2} \\
& +c_{n, m}\left\|y_{n, m-1}-q\right\|^{2}-a_{n, m} c_{n, m}\left\|u_{n}-z_{n, m}\right\|^{2} \\
\leq & \left\|u_{n}-q\right\|^{2}-a_{n, m} c_{n, m}\left\|u_{n}-z_{n, m}\right\|^{2} \\
& -a_{n, m-1} c_{n, m-1} c_{n, m}\left\|u_{n}-z_{n, m-1}\right\|^{2} \\
& -\ldots-a_{n, 1} c_{n, 1} c_{n, 2} \ldots c_{n, m}\left\|u_{n}-z_{n, 1}\right\|^{2} \\
& -a_{n, 1} b_{n, 1} c_{n, 2} \ldots c_{n, m}\left\|u_{n}-x_{n}\right\|^{2}, \\
= &
\end{aligned}
$$


which implies that

$$
\begin{aligned}
\left\|x_{n+1}-q\right\|^{2}= & \left\|\vartheta_{n} f x_{n}+\left(1-\vartheta_{n}\right) y_{n, m}-q\right\|^{2} \\
\leq & \vartheta_{n}\left\|f x_{n}-q\right\|^{2}+\left(1-\vartheta_{n}\right)\left\|y_{n, m}-q\right\|^{2} \\
\leq & \vartheta_{n}\left\|f x_{n}-q\right\|^{2}+\left(1-\vartheta_{n}\right)\left\|u_{n}-q\right\|^{2} \\
& -\left(1-\vartheta_{n}\right) a_{n, m} c_{n, m}\left\|u_{n}-z_{n, m}\right\|^{2} \\
& -\left(1-\vartheta_{n}\right) a_{n, m-1} c_{n, m-1} c_{n, m}\left\|u_{n}-z_{n, m-1}\right\|^{2} \\
& -\cdots-\left(1-\vartheta_{n}\right) a_{n, 1} c_{n, 1} c_{n, 2} \ldots c_{n, m}\left\|u_{n}-z_{n, 1}\right\|^{2} \\
& -\left(1-\vartheta_{n}\right) a_{n, 1} b_{n, 1} c_{n, 2} \ldots c_{n, m}\left\|u_{n}-x_{n}\right\|^{2} .
\end{aligned}
$$

Therefore, we have that

$$
\begin{aligned}
& \left(1-\vartheta_{n}\right) a_{n, 1} b_{n, 1} c_{n, 2} \ldots c_{n, m}\left\|u_{n}-x_{n}\right\|^{2} \\
& \quad \leq\left\|x_{n}-q\right\|^{2}-\left\|x_{n+1}-q\right\|^{2}+\vartheta_{n}\left\|\gamma f x_{n}-q\right\| .
\end{aligned}
$$

In order to prove that $x_{n} \rightarrow q$ as $n \rightarrow \infty$, we consider the following two cases.

Case 1. Suppose that there exists $n_{0}$ such that $\left\{\left\|x_{n}-q\right\|\right\}$ is nonincreasing, for all $n \geq n_{0}$. Boundedness of $\left\{\left\|x_{n}-q\right\|\right\}$ implies that $\left\|x_{n}-q\right\|$ is convergent. From (31) and conditions (i), (ii) we have that

$$
\lim _{n \rightarrow \infty}\left\|u_{n}-x_{n}\right\|=0
$$

By a similar argument, for $k=1,2, \ldots, m$, we obtain that

$$
\lim _{n \rightarrow \infty}\left\|u_{n}-z_{n, k}\right\|=0
$$

Hence,

$$
\begin{array}{r}
\lim _{n \rightarrow \infty} \operatorname{dist}\left(u_{n}, T_{1} u_{n}\right) \leq \lim _{n \rightarrow \infty}\left\|u_{n}-z_{n, 1}\right\|=0, \\
\lim _{n \rightarrow \infty} \operatorname{dist}\left(u_{n}, T_{k} y_{n, k-1}\right) \leq \lim _{n \rightarrow \infty}\left\|u_{n}-z_{n, k}\right\|=0,
\end{array}
$$

Therefore, we have

$$
\begin{aligned}
\lim _{n \rightarrow \infty}\left\|u_{n}-y_{n, 1}\right\| \leq & \lim _{n \rightarrow \infty} b_{n, 1}\left\|u_{n}-x_{n}\right\| \\
& +\lim _{n \rightarrow \infty} c_{n, 1}\left\|u_{n}-z_{n, 1}\right\|=0 .
\end{aligned}
$$

For $k=2, \ldots, m$, we have

$$
\begin{aligned}
\lim _{n \rightarrow \infty}\left\|u_{n}-y_{n, k}\right\| \leq & \lim _{n \rightarrow \infty} b_{n, k}\left\|u_{n}-z_{n, k-1}\right\| \\
& +\lim _{n \rightarrow \infty} c_{n, k}\left\|u_{n}-z_{n, k}\right\|=0 .
\end{aligned}
$$

Using the previous inequality for $k=2, \ldots, m$, we have

$$
\begin{aligned}
& \operatorname{dist}\left(u_{n}, T_{k} u_{n}\right) \leq \operatorname{dist}\left(u_{n}, T_{k} y_{n, k-1}\right)+H\left(T_{k} y_{n, k-1}, T_{k} u_{n}\right) \\
& \leq \operatorname{dist}\left(u_{n}, T_{k} y_{n, k-1}\right)+\mu \operatorname{dist}\left(y_{n, k-1}, T_{k} y_{n, k-1}\right) \\
& \quad+\left\|y_{n, k-1}-u_{n}\right\| \\
& \leq(\mu+1) \operatorname{dist}\left(u_{n}, T_{k} y_{n, k-1}\right)+(\mu+1)\left\|y_{n, k-1}-u_{n}\right\| \\
& \leq(\mu+1)\left\|u_{n}-z_{n, k}\right\|+(\mu+1)\left\|y_{n, k-1}-u_{n}\right\| \longrightarrow 0, \\
& n \longrightarrow \infty .
\end{aligned}
$$

Next, we show that

$$
\limsup _{n \rightarrow \infty}\left\langle q-f q, q-x_{n}\right\rangle \leq 0,
$$

where $q=P_{\mathscr{F}} f(q)$. To show this inequality, we choose a subsequence $\left\{x_{n_{i}}\right\}$ of $\left\{x_{n}\right\}$ such that

$$
\lim _{i \rightarrow \infty}\left\langle q-f q, q-x_{n_{i}}\right\rangle=\limsup _{n \rightarrow \infty}\left\langle q-f q, q-x_{n}\right\rangle .
$$

Since $\left\{x_{n_{i}}\right\}$ is bounded, there exists a subsequence $\left\{x_{n_{i_{j}}}\right\}$ of $\left\{x_{n_{i}}\right\}$ which converges weakly to $v$. Without loss of generality, we can assume that $x_{n_{i}}$ converges weakly to $v$. Since $\lim _{n \rightarrow \infty}\left\|x_{n}-u_{n}\right\|=0$, we have $u_{n_{i}}$ converges weakly to $v$. We show that $v \in \mathscr{F}$. Let us show $v \in E P(\Psi)$. Since $u_{n}=S_{r_{n}} x_{n}$, we have

$$
\Psi\left(u_{n}, y\right)+\frac{1}{r_{n}}\left\langle y-u_{n}, u_{n}-x_{n}\right\rangle \geq 0 \quad \forall y \in C .
$$

From (A2), we have

$$
\frac{1}{r_{n}}\left\langle y-u_{n}, u_{n}-x_{n}\right\rangle \geq \Psi\left(y, u_{n}\right) .
$$

Replacing $n$ with $n_{i}$, we have

$$
\left\langle y-u_{n_{i}}, \frac{u_{n_{i}}-x_{n_{i}}}{r_{n_{i}}}\right\rangle \geq \Psi\left(y, u_{n_{i}}\right) .
$$

From (A4), we have

$$
0 \geq \Psi(y, v), \quad \forall y \in C .
$$

For $t \in(0,1]$ and $y \in C$, let $y_{t}=t y+(1-t) v$. Since $y, v \in C$, and $C$ is convex, we have $y_{t} \in C$ and hence $\Psi\left(y_{t}, v\right) \leq 0$. So, from (A1) and (A4) we have

$$
0=\Psi\left(y_{t}, y_{t}\right) \leq t \Psi\left(y_{t}, y\right)+(1-t) \Psi\left(y_{t}, v\right) \leq t \Psi\left(y_{t}, y\right),
$$

which gives $0 \leq \Psi\left(y_{t}, y\right)$. Letting $t \rightarrow 0$, we have, for each $y \in C, 0 \leq \Psi(v, y)$ Also, since $u_{n_{i}} \rightarrow v$ and $\lim _{n \rightarrow \infty} \operatorname{dist}\left(u_{n}, T_{i} u_{n}\right)=0$, by Lemma 12 we have $v \in$ $\bigcap_{i=1}^{m} F\left(T_{i}\right)$. Hence, $v \in \mathscr{F}$. Since $q=P_{\mathscr{F}} f(q)$ and $v \in \mathscr{F}$, it follows that

$$
\begin{gathered}
\limsup _{n \rightarrow \infty}\left\langle q-f q, q-x_{n}\right\rangle=\lim _{i \rightarrow \infty}\left\langle q-f q, q-x_{n_{i}}\right\rangle \\
=\langle q-f q, q-v\rangle \leq 0 .
\end{gathered}
$$


By using Lemma 7 and inequality (31) we have

$$
\begin{aligned}
&\left\|x_{n+1}-q\right\|^{2} \\
& \leq\left\|\left(1-\vartheta_{n}\right)\left(y_{n, m}-q\right)\right\|^{2}+2 \vartheta_{n}\left\langle f x_{n}-q, x_{n+1}-q\right\rangle \\
& \leq\left(1-\vartheta_{n}\right)^{2}\left\|y_{n, m}-q\right\|^{2}+2 \vartheta_{n}\left\langle f x_{n}-f q, x_{n+1}-q\right\rangle \\
&+2 \vartheta_{n}\left\langle f q-q, x_{n+1}-q\right\rangle \\
& \leq\left(1-\vartheta_{n}\right)^{2}\left\|x_{n}-q\right\|^{2}+2 \vartheta_{n} k\left\|x_{n}-q\right\|\left\|x_{n+1}-q\right\| \\
&+2 \vartheta_{n}\left\langle f q-q, x_{n+1}-q\right\rangle \\
& \leq\left(1-\vartheta_{n}\right)^{2}\left\|x_{n}-q\right\|^{2}+\vartheta_{n} k\left(\left\|x_{n}-q\right\|^{2}+\left\|x_{n+1}-q\right\|^{2}\right) \\
&+2 \vartheta_{n}\left\langle f q-q, x_{n+1}-q\right\rangle \\
& \leq\left(\left(1-\vartheta_{n}\right)^{2}+\vartheta_{n} k\right)\left\|x_{n}-q\right\|^{2}+\vartheta_{n} k\left\|x_{n+1}-q\right\|^{2} \\
&+2 \vartheta_{n}\left\langle f q-q, x_{n+1}-q\right\rangle .
\end{aligned}
$$

This implies that

$$
\begin{aligned}
\left\|x_{n+1}-q\right\|^{2} \leq & \left(1-\frac{2(1-k) \vartheta_{n}}{1-\vartheta_{n} k}\right)\left\|x_{n}-q\right\|^{2} \\
& +\frac{\vartheta_{n}^{2}}{1-\vartheta_{n} k}\left\|x_{n}-q\right\|^{2} \\
& +\frac{2 \vartheta_{n}}{1-\vartheta_{n} k}\left\langle f q-q, x_{n+1}-q\right\rangle .
\end{aligned}
$$

From Lemma 8, we conclude that the sequence $\left\{x_{n}\right\}$ converges strongly to $q$.

Case 2. Assume that there exists a subsequence $\left\{x_{n_{j}}\right\}$ of $\left\{x_{n}\right\}$ such that

$$
\left\|x_{n_{j}}-q\right\|<\left\|x_{n_{j+1}}-q\right\|,
$$

for all $j \in \mathbb{N}$. In this case, from Lemma 9, there exists a nondecreasing sequence $\{\tau(n)\}$ of $\mathbb{N}$ for all $n \geq n_{0}$ (for some $n_{0}$ large enough) such that $\tau(n) \rightarrow \infty$ as $n \rightarrow \infty$ and the following inequalities hold for all $n \geq n_{0}$ :

$$
\left\|x_{\tau(n)}-q\right\| \leq\left\|x_{\tau(n)+1}-q\right\|, \quad\left\|x_{n}-q\right\| \leq\left\|x_{\tau(n)+1}-q\right\| .
$$

From (31) we obtain $\lim _{n \rightarrow \infty}\left\|u_{\tau(n)}-T_{i} u_{\tau(n)}\right\|=0$, and $\lim _{n \rightarrow \infty}\left\|u_{\tau(n)}-x_{\tau(n)}\right\|=0$. Following an argument similar to that in Case 1 , we have

$$
\lim _{n \rightarrow \infty}\left\|x_{\tau(n)}-q\right\|=0, \quad \lim _{n \rightarrow \infty}\left\|x_{\tau(n)+1}-q\right\|=0 .
$$

Thus, by Lemma 9 we have

$$
0 \leq\left\|x_{n}-q\right\| \leq \max \left\{\left\|x_{\tau(n)}-q\right\|,\left\|x_{n}-q\right\|\right\} \leq\left\|x_{\tau(n)+1}-q\right\| .
$$

Therefore, $\left\{x_{n}\right\}$ converges strongly to $q=P_{\mathscr{F}} f(q)$. This completes the proof.
Now, we remove the condition that $T(p)=\{p\}$ for all $p \in$ $\mathscr{F}$, and state the following theorem.

Theorem 14. Let $C$ be a nonempty closed convex subset of a real Hilbert space $H$ and $\Psi$ a bifunction of $C \times C$ into $\mathbb{R}$ satisfying $(A 1)-(A 4)$. Let, for each $1 \leq i \leq m, T_{i}: C \rightarrow P(C)$ be multivalued mappings such that $P_{T_{i}}$ satisfies the condition (E). Assume that $\mathscr{F}=\bigcap_{i=1}^{m} F\left(T_{i}\right) \bigcap E P(\Psi) \neq \emptyset$. Let $f$ be a $k$-contraction of $C$ into itself. Let $\left\{x_{n}\right\}$ and $\left\{u_{n}\right\}$ be sequences generated the following algorithm:

$$
\begin{gathered}
x_{0} \in C, \\
u_{n} \in C \text { such that } \Psi\left(u_{n}, y\right)+\frac{1}{r_{n}}\left\langle y-u_{n}, u_{n}-x_{n}\right\rangle \geq 0, \\
y_{n, 1}=a_{n, 1} u_{n}+b_{n, 1} x_{n}+c_{n, 1} z_{n, 1}, \quad \forall y \in C \\
y_{n, 2}=a_{n, 2} u_{n}+b_{n, 2} z_{n, 1}+c_{n, 2} z_{n, 2}, \\
y_{n, 3}=a_{n, 3} u_{n}+b_{n, 3} z_{n, 2}+c_{n, 3} z_{n, 3} \\
\vdots \\
y_{n, m}=a_{n, m} u_{n}+b_{n, m} z_{n, m-1}+c_{n, m} z_{n, m}, \\
x_{n+1}=\vartheta_{n} f x_{n}+\left(1-\vartheta_{n}\right) y_{n, m}, \quad \forall n \geq 0,
\end{gathered}
$$

where $z_{n, 1} \in P_{T_{1}}\left(u_{n}\right), z_{n, k} \in P_{T_{k}}\left(y_{n, k-1}\right)$ for $k=2, \ldots, m$, and $\left\{a_{n, i}\right\},\left\{b_{n, i}\right\},\left\{c_{n, i}\right\},\left\{\vartheta_{n}\right\}$ and, $\left\{r_{n}\right\}$ satisfy the following conditions:

(i) $\left\{a_{n, i}\right\},\left\{b_{n, i}\right\},\left\{c_{n, i}\right\} \subset[a, b] \subset(0,1), a_{n, i}+b_{n, i}+c_{n, i}=$ $1,(i=1,2, \ldots, m)$,

(ii) $\left\{\vartheta_{n}\right\} \subset(0,1), \lim _{n \rightarrow \infty} \vartheta_{n}=0, \sum_{n=1}^{\infty} \vartheta_{n}=\infty$,

(iii) $\left\{r_{n}\right\} \subset(0, \infty)$, and $\lim \inf _{n \rightarrow \infty} r_{n}>0$.

Then, the sequences $\left\{x_{n}\right\}$ and $\left\{u_{n}\right\}$ converge strongly to $q \in \mathscr{F}$, where $q=P_{\mathscr{F}} f(q)$.

Proof. Let $p \in \mathscr{F}$; then $P_{T_{i}}(p)=\{p\},(i=1,2, \ldots, m)$. Now by substituting $P_{T_{i}}$ instead of $T_{i}$, and using a similar argument as in the proof of Theorem 13, the desired result follows.

As a corollary for single-valued mappings, we obtain the following result.

Corollary 15. Let $C$ be a nonempty closed convex subset of a real Hilbert space $H$ and $\Psi$ a bifunction of $C \times C$ into $\mathbb{R}$ satisfying (A1)-(A4). Let, for each $1 \leq i \leq m, T_{i}: C \rightarrow C$ be a finite family of mappings satisfying condition $(E)$. Assume that $\mathscr{F}=\bigcap_{i=1}^{m} F\left(T_{i}\right) \bigcap E P(\Psi) \neq \emptyset$. Let $f$ be a $k$-contraction 
of $C$ into itself. Let $\left\{x_{n}\right\}$ and $\left\{u_{n}\right\}$ be sequences generated the following algorithm:

$$
\begin{gathered}
x_{0} \in C, \\
u_{n} \in C \text { such that } \Psi\left(u_{n}, y\right)+\frac{1}{r_{n}}\left\langle y-u_{n}, u_{n}-x_{n}\right\rangle \geq 0, \\
y_{n, 1}=a_{n, 1} u_{n}+b_{n, 1} x_{n}+c_{n, 1} T_{1} u_{n}, \quad \forall y \in C \\
y_{n, 2}=a_{n, 2} u_{n}+b_{n, 2} T_{1} u_{n}+c_{n, 2} T_{2} y_{n, 1} \\
\vdots \\
y_{n, m}=a_{n, m} u_{n}+b_{n, m} T_{m-1} y_{n, m-2}+T_{m} y_{n, m-1}, \\
x_{n+1}=\vartheta_{n} f x_{n}+\left(1-\vartheta_{n}\right) y_{n, m}, \quad \forall n \geq 0,
\end{gathered}
$$

where $\left\{a_{n, i}\right\},\left\{b_{n, i}\right\},\left\{c_{n, i}\right\},\left\{\vartheta_{n}\right\}$, and $\left\{r_{n}\right\}$ satisfy the following conditions:

(i) $\left\{a_{n, i}\right\},\left\{b_{n, i}\right\},\left\{c_{n, i}\right\} \subset[a, b] \subset(0,1), a_{n, i}+b_{n, i}+c_{n, i}=1$, $(i=1,2, \ldots, m)$,

(ii) $\left\{\vartheta_{n}\right\} \subset(0,1), \lim _{n \rightarrow \infty} \vartheta_{n}=0, \sum_{n=1}^{\infty} \vartheta_{n}=\infty$

(iii) $\left\{r_{n}\right\} \subset(0, \infty)$, and $\lim _{\inf _{n \rightarrow \infty}} r_{n}>0$.

Then, the sequences $\left\{x_{n}\right\}$ and $\left\{u_{n}\right\}$ converge strongly to $q \in \mathscr{F}$, where $q=P_{\mathscr{F}} f(q)$.

Remark 16. Our results generalize the corresponding results of S. Takahashi and W. Takahashi [9] from a single valued nonexpansive mapping to a finite family of multivalued mappings satisfying the condition $(E)$. Our results also improve the recent results of Eslamian [16].

\section{Conflict of Interests}

The authors declare that there is no conflict of interests regarding the publication of this paper.

\section{Acknowledgments}

This paper was funded by the Deanship of Scientific Research (DSR), King Abdulaziz University, Jeddah. The authors therefore, acknowledge with thanks DSR for technical and financial support. The authors are also thankful to the referees for their valuable suggestion/comments.

\section{References}

[1] J. García-Falset, E. Llorens-Fuster, and T. Suzuki, "Fixed point theory for a class of generalized nonexpansive mappings," Journal of Mathematical Analysis and Applications, vol. 375, no. 1, pp. 185-195, 2011.

[2] A. Abkar and M. Eslamian, "Common fixed point results in CAT(0) spaces," Nonlinear Analysis: Theory, Methods \& Applications, vol. 74, no. 5, pp. 1835-1840, 2011.
[3] M. Eslamian and A. Abkar, "Fixed point theorems for suzuki generalized nonexpansive multivalued mappings in Banach spaces," Fixed Point Theory and Applications, vol. 2010, Article ID 457935, 10 pages, 2010.

[4] E. Blum and W. Oettli, "From optimization and variational inequalities to equilibrium problems," The Mathematics Student, vol. 63, no. 1-4, pp. 123-145, 1994.

[5] S. D. Flåm and A. S. Antipin, "Equilibrium programming using proximal-like algorithms," Mathematical Programming, vol. 78, no. 1, Ser. A, pp. 29-41, 1997.

[6] P. L. Combettes and S. A. Hirstoaga, "Equilibrium programming in Hilbert spaces," Journal of Nonlinear and Convex Analysis, vol. 6, no. 1, pp. 117-136, 2005.

[7] A. Latif, L.-C. Ceng, and Q. H. Ansari, "Multi-step hybrid viscosity method for systems of variational inequalities defined over sets of solutions of an equilibrium problem and fixed point problems," Fixed Point Theory and Applications, vol. 2012, article 186, 26 pages, 2012.

[8] A. Tada and W. Takahashi, "Weak and strong convergence theorems for a nonexpansive mapping and an equilibrium problem," Journal of Optimization Theory and Applications, vol. 133, no. 3, pp. 359-370, 2007.

[9] S. Takahashi and W. Takahashi, "Viscosity approximation methods for equilibrium problems and fixed point problems in Hilbert spaces," Journal of Mathematical Analysis and Applications, vol. 331, no. 1, pp. 506-515, 2007.

[10] L.-C. Ceng, S. Al-Homidan, Q. H. Ansari, and J.-C. Yao, "An iterative scheme for equilibrium problems and fixed point problems of strict pseudo-contraction mappings," Journal of Computational and Applied Mathematics, vol. 223, no. 2, pp. 967-974, 2009.

[11] L.-C. Ceng, Q. H. Ansari, and J.-C. Yao, "Viscosity approximation methods for generalized equilibrium problems and fixed point problems," Journal of Global Optimization, vol. 43, no. 4, pp. 487-502, 2009.

[12] L.-C. Zeng, Q. H. Ansari, and S. Al-Homidan, "Hybrid proximal-type algorithms for generalized equilibrium problems, maximal monotone operators, and relatively nonexpansive mappings," Fixed Point Theory and Applications, vol. 2011, Article ID 973028, 23 pages, 2011.

[13] S. Plubtieng and R. Punpaeng, "A general iterative method for equilibrium problems and fixed point problems in Hilbert spaces," Journal of Mathematical Analysis and Applications, vol. 336, no. 1, pp. 455-469, 2007.

[14] L.-C. Ceng, N. Hadjisavvas, and N.-C. Wong, "Strong convergence theorem by a hybrid extragradient-like approximation method for variational inequalities and fixed point problems," Journal of Global Optimization, vol. 46, no. 4, pp. 635-646, 2010.

[15] F. Cianciaruso, G. Marino, and L. Muglia, "Iterative methods for equilibrium and fixed point problems for nonexpansive semigroups in Hilbert spaces," Journal of Optimization Theory and Applications, vol. 146, no. 2, pp. 491-509, 2010.

[16] M. Eslamian, "Convergence theorems for nonspreading mappings and nonexpansive multivalued mappings and equilibrium problems," Optimization Letters, vol. 7, no. 3, pp. 547-557, 2013.

[17] M. Eslamian, "Hybrid method for equilibrium problems and fixed point problems of finite families of nonexpansive semigroups," Revista de la Real Academia de Ciencias Exactas, Fisicas y Naturales. Serie A, vol. 107, no. 2, pp. 299-307, 2013.

[18] A. Moudafi, "Weak convergence theorems for nonexpansive mappings and equilibrium problems," Journal of Nonlinear and Convex Analysis, vol. 9, no. 1, pp. 37-43, 2008. 
[19] U. Kamraksa and R. Wangkeeree, "Generalized equilibrium problems and fixed point problems for nonexpansive semigroups in Hilbert spaces," Journal of Global Optimization, vol. 51, no. 4, pp. 689-714, 2011.

[20] A. Abkar and M. Eslamian, "Strong convergence theorems for equilibrium problems and fixed point problem of multivalued nonexpansive mappings via hybrid projection method," Journal of Inequalities and Applications, vol. 2012, article 164, 13 pages, 2012.

[21] S. Wang, G. Marino, and Y.-C. Liou, "Strong convergence theorems for variational inequality, equilibrium and fixed point problems with applications," Journal of Global Optimization, vol. 54, no. 1, pp. 155-171, 2012.

[22] X. Qin, Y. J. Cho, and S. M. Kang, "Viscosity approximation methods for generalized equilibrium problems and fixed point problems with applications," Nonlinear Analysis: Theory, Methods \& Applications, vol. 72, no. 1, pp. 99-112, 2010.

[23] C. Byrne, "A unified treatment of some iterative algorithms in signal processing and image reconstruction," Inverse Problems, vol. 20, no. 1, pp. 103-120, 2004.

[24] C. I. Podilchuk and R. J. Mammone, "Image recovery by convex projections using a least-squares constraint," Journal of the Optical Society of America A, vol. 7, pp. 517-521, 1990.

[25] D. C. Youla, "On deterministic convergence of iterations of relaxed projection operators," Journal of Visual Communication and Image Representation, vol. 1, no. 1, pp. 12-20, 1990.

[26] W. R. Mann, "Mean value methods in iteration," Proceedings of the American Mathematical Society, vol. 4, pp. 506-510, 1953.

[27] S. Ishikawa, "Fixed points by a new iteration method," Proceedings of the American Mathematical Society, vol. 44, pp. 147-150, 1974.

[28] R. P. Agarwal, D. O’Regan, and D. R. Sahu, "Iterative construction of fixed points of nearly asymptotically nonexpansive mappings," Journal of Nonlinear and Convex Analysis, vol. 8, no. 1, pp. 61-79, 2007.

[29] E. Lami Dozo, "Multivalued nonexpansive mappings and Opial's condition," Proceedings of the American Mathematical Society, vol. 38, pp. 286-292, 1973.

[30] T. C. Lim, "A fixed point theorem for multivalued nonexpansive mappings in a uniformly convex Banach space," Bulletin of the American Mathematical Society, vol. 80, pp. 1123-1126, 1974.

[31] S. Dhompongsa, A. Kaewkhao, and B. Panyanak, "Browder's convergence theorem for multivalued mappings without endpoint condition," Topology and Its Applications, vol. 159, no. 1011, pp. 2757-2763, 2012.

[32] M. A. Khamsi and W. A. Kirk, "On uniformly Lipschitzian multivalued mappings in Banach and metric spaces," Nonlinear Analysis: Theory, Methods \& Applications, vol. 72, no. 3-4, pp. 2080-2085, 2010.

[33] J. Garcia-Falset, E. Llorens-Fuster, and E. Moreno-Galvez, "Fixed point theory for multivalued generalized nonexpansive mappings," Applicable Analysis and Discrete Mathematics, vol. 6, no. 2, pp. 265-286, 2012.

[34] A. Latif, T. Husain, and I. Beg, "Fixed point of nonexpansive type and K-multivalued maps," International Journal of Mathematics and Mathematical Sciences, vol. 17, no. 3, pp. 429-435, 1994.

[35] S. Dhompongsa, W. A. Kirk, and B. Panyanak, "Nonexpansive set-valued mappings in metric and Banach spaces," Journal of Nonlinear and Convex Analysis, vol. 8, no. 1, pp. 35-45, 2007.
[36] A. Abkar and M. Eslamian, "Fixed point theorems for Suzuki generalized nonexpansive multivalued mappings in Banach spaces," Fixed Point Theory and Applications, vol. 2010, Article ID 457935, 10 pages, 2010.

[37] Y. Song and H. Wang, "Convergence of iterative algorithms for multivalued mappings in Banach spaces," Nonlinear Analysis: Theory, Methods \& Applications, vol. 70, no. 4, pp. 1547-1556, 2009.

[38] A. Latif and I. Beg, "Geometric fixed points for single and multivalued mappings," Demonstratio Mathematica, vol. 30, no. 4, pp. 791-800, 1997.

[39] M. Eslamian and A. Abkar, "One-step iterative process for a finite family of multivalued mappings," Mathematical and Computer Modelling, vol. 54, no. 1-2, pp. 105-111, 2011.

[40] S. Dhompongsa, A. Kaewkhao, and B. Panyanak, “On Kirk's strong convergence theorem for multivalued nonexpansive mappings on CAT(0) spaces," Nonlinear Analysis: Theory, Methods \& Applications, vol. 75, no. 2, pp. 459-468, 2012.

[41] M. O. Osilike and D. I. Igbokwe, "Weak and strong convergence theorems for fixed points of pseudocontractions and solutions of monotone type operator equations," Computers \& Mathematics with Applications, vol. 40, no. 4-5, pp. 559-567, 2000.

[42] H. K. Xu, "An iterative approach to quadratic optimization," Journal of Optimization Theory and Applications, vol. 116, no. 3, pp. 659-678, 2003.

[43] P.-E. Maingé, "Strong convergence of projected subgradient methods for nonsmooth and nonstrictly convex minimization," Set-Valued Analysis, vol. 16, no. 7-8, pp. 899-912, 2008. 


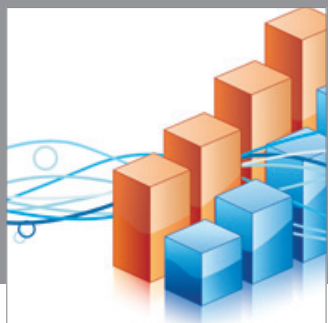

Advances in

Operations Research

mansans

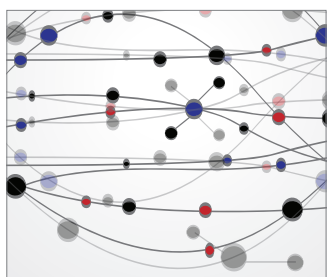

The Scientific World Journal
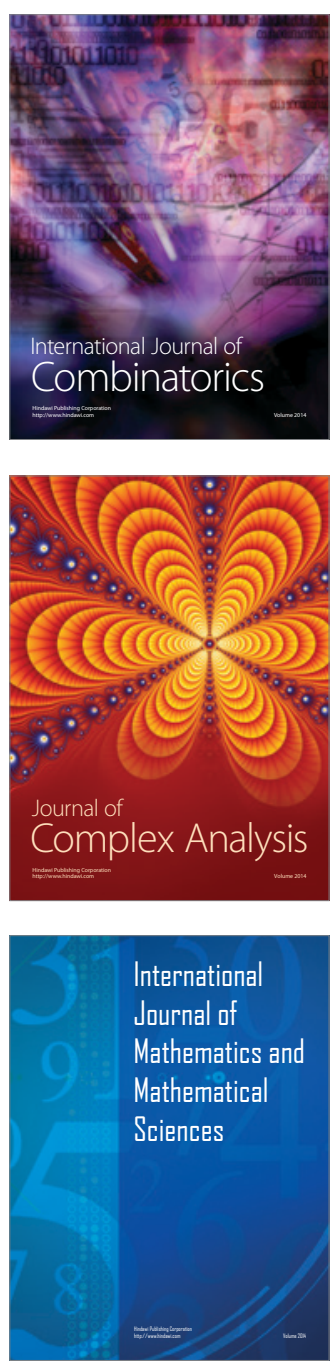
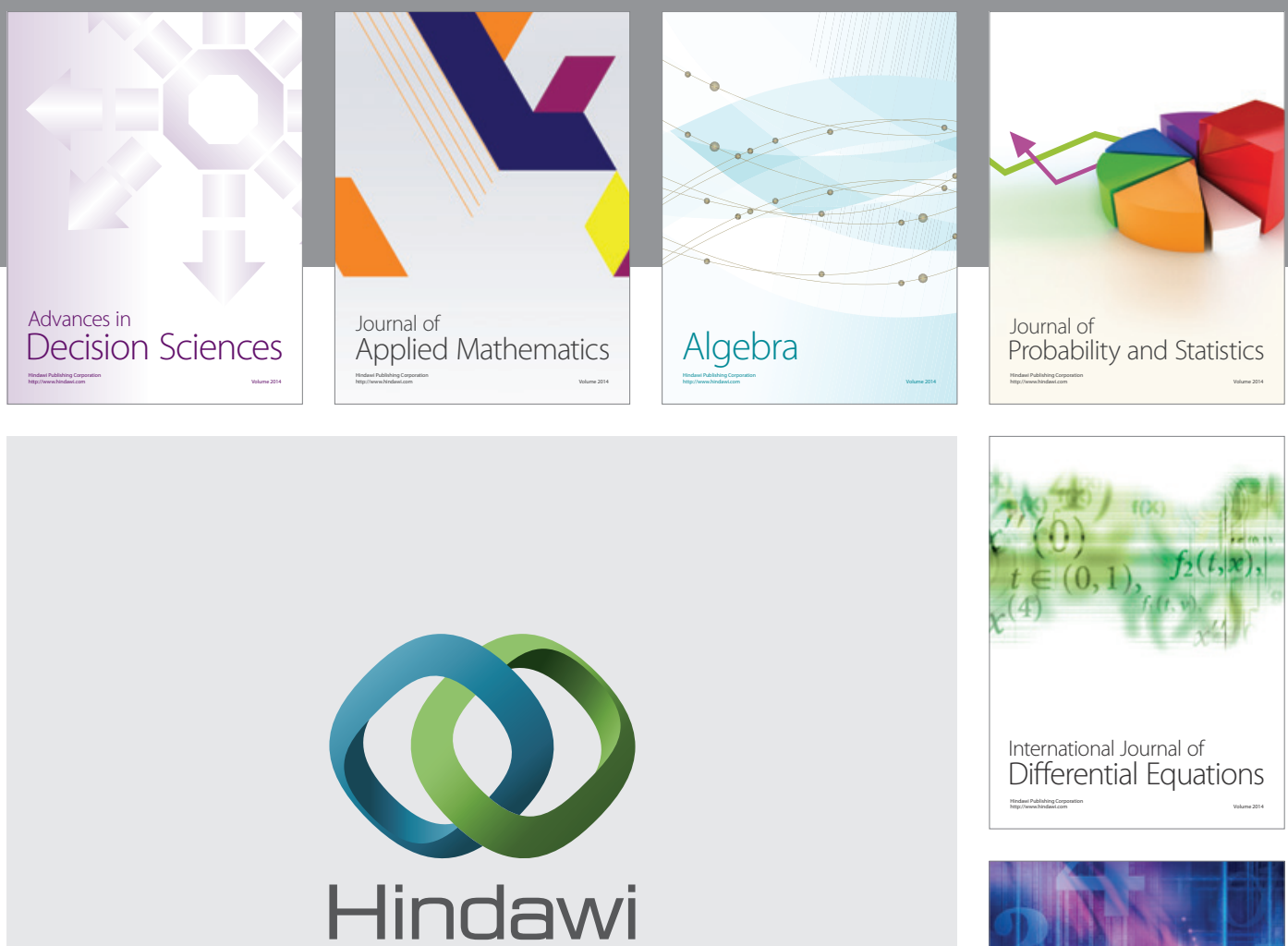

Submit your manuscripts at http://www.hindawi.com
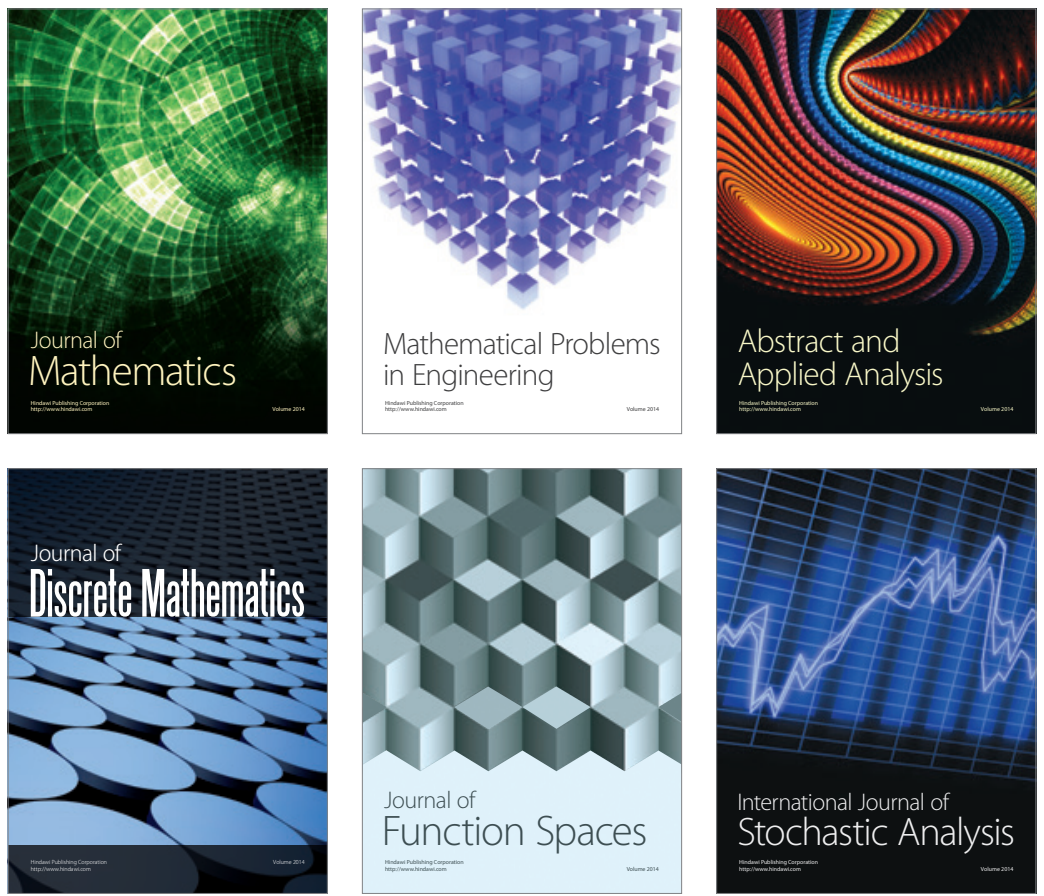

Journal of

Function Spaces

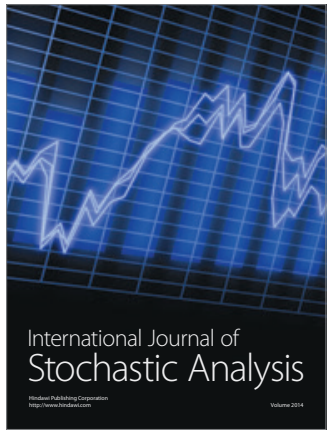

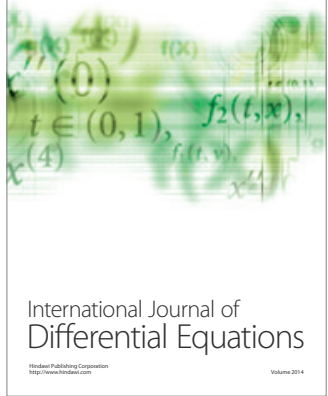
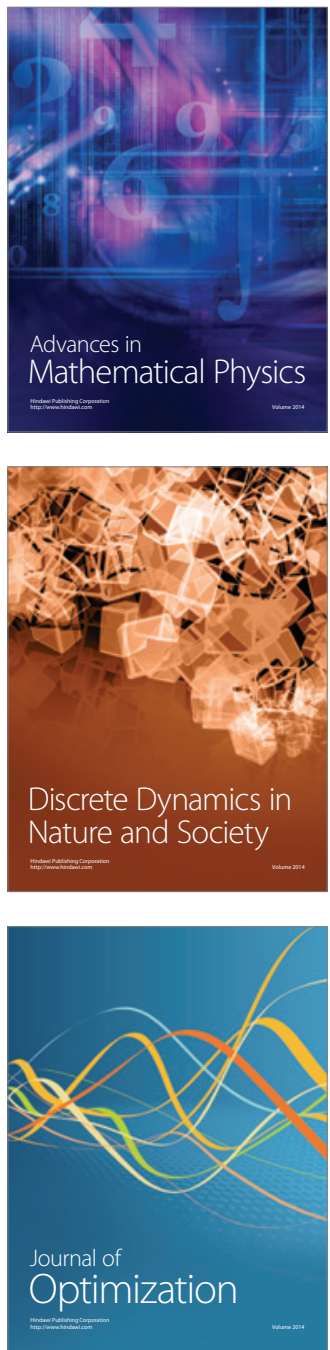